\title{
Clinical features and long-term follow-up of quasi-moyamoya disease in children
}

Kentaro Hayashi, Nobutaka Horie, Kazuhiko Suyama, Izumi Nagata

Department of Neurosurgery, Nagasaki University School of Medicine, Nagasaki, Japan

Correspondence to Kentaro Hayashi M.D.

1-7-1 Sakamoto, Nagasaki-city 852-8501 Japan

Tel; +81-95-849-7375

Fax; +81-95-849-7378

e-mail; kenkuni@net.nagasaki-u.ac.jp

Short title: Clinical features of quasi-moyamoya disease 


\section{Abstract}

Background: A inherited or acquired disorders and conditions may present in conjunction with moyamoya disease. This condition is known as quasi-moyamoya disease. Methods: A retrospective review of 69 moyamoya disease patients treated for the past 20 years identified seven patients of quasi-moyamoya disease and five of them were pediatric patients. Results: The mean age at initial diagnosis was 6.4 year-old (range 5 to 9). Associated disorders were as follows; craniosynostosis, dwarfism with coarctation of aorta, Proteus syndrome, cranial irradiation for brain tumor. Their clinical type included cerebral ischemia in three patients, cerebral bleeding with ischemia in one and epilepsy in one. Three patients with cerebral ischemia underwent bypass surgery and their ischemia was improved. One patient died of brain tumor recurrence and the activity of daily living was affected by mental retardation due to associated disorder. Conclusions: The clinical course and radiological finding of quasi-moyamoya disease are diverse because of associated disorders, distinguishing definite moyamoya disease.

Key word: ischemic stroke, moyamoya disease, brain tumor, radiation therapy 


\section{Introduction}

Moyamoya disease is characterized by the progressive occlusion of the internal carotid artery (ICA) or its terminal branches, accompanied by the formation of extensive collateral vessels (moyamoya vessels) at the base of the brain. This disease has been reported in association with various disease entities including atherosclerosis, autoimmune disease, meningitis, brain tumor, neurofibromatosis type 1, Down syndrome, cranial irradiation and so on [1-3]. These conditions are distinguished from moyamoya disease according to the diagnostic criteria of the Research Committee on Moyamoya Disease (Spontaneous Occlusion of the Circle of Willis) of Ministry of Health and Welfare of Japan (RCMJ), and named as quasi-moyamoya disease [4, 5]. Since the incidence of quasi-moyamoya disease is quite rare, the clinical course has been unclear and the consensus for the treatment has not been established. We retrospectively reviewed our experience of quasi-moyamoya disease, and the clinical features and surgical management of quasi-moyamoya disease are described. 


\section{Materials and Methods}

Moyamoya disease patients diagnosed at Nagasaki University Hospital from 1985 to 2005 were retrospectively reviewed. Fifty-two patients were diagnosed as definite moyamoya disease based on the guidelines for the diagnosis of moyamoya disease set by RCMJ. Unilateral moyamoya disease (probable moyamoya disease) and quasi-moyamoya disease was defined angiographically and identified in 9 and 7 patients respectively. The associated disorders of quasi-moyamoya disease were craniosynostosis, neurofibromatosis type 1 , dwarfism with coarctation of aorta, Proteus syndrome, cranial irradiation for brain tumor and atherosclerosis. Among 7 patients of quasi-moyamoya disease, 5 patients were less than 15-years-old. We analyzed medical history, age at onset, first sign at onset angiographic stage (Suzuki classification), ischemic or hemorrhagic lesions on CT or MRI, findings of hemodynamic hypoperfusion or decreased vascular reactivity to acetazolamide on single photon emission tomography (SPECT). Thus, surgical treatment was attempted when severe cerebral hypoperfusion was revealed. Patients were followed clinically and radiologically. When the progressing of the occlusive lesion in the major intracranial arteries was suspected, angiography was performed to verify it. 


\section{Results}

Baseline patient characteristics of quasi-moyamoya disease were summarized in table 1 . The male-to-female ratio was 4 to 1 , and the mean age at initial diagnosis was 6.4 year-old (range 5 to 9). Their clinical symptoms were complicated because preexisted neurological symptoms and acquired disorders were mixed. Clinical classification included ischemic type in three patients, bleeding with ischemia type in one, epilepsy in one. Mental retardation had been found in four patients and visual disturbance was identified in one case of brain tumor. Angiographically, all patient had bilateral lesion. The initial angiographic stages by Suzuki were grade III $(n=4)$ or IV $(n=1)$. Thus, stenosis was found in the terminal portion of the ICA or the horizontal portion of the middle cerebral artery (MCA). Moyamoya vessels were seen bilaterally in all patients. The collateral flow from external carotid artery was well developed in two cranial irradiation patients.

Three patients underwent vascular reconstruction namely encephalo-duro-arterio-synostosis (EDAS) or superficial temporal artery (STA)-MCA anastomosis combination with encephalo-myo-synangiosis (EMS). Postoperative courses were uneventful and the ischemic attack has disappeared. Vascular reconstruction was not employed for two cases of brain tumor, since collateral flow from external carotid artery had been developed. The patients have been followed by noting clinical symptoms and by SPECT, MRI including MR angiography. Mean follow-up duration was 11.0 years, ranging 3 to 20 years. Angiographical disease 
progression was observed in two cases of cranial irradiation for brain tumor. One patient died of brain tumor recurrence and the other 4 patients of daily life was influenced with mental retardation due to associated disorder. No one exhibited familial moyamoya disease.

\section{Illustrative Cases}

\section{Patient 1}

A 6-year-old boy who had craniosynostosis (Fig. 1A) and mental retardation, and had been diagnosed as Saethre-Chotzen syndrome presented because of gait disturbance. MRI showed multiple lacuna infarctions (Fig. 1B). Cerebral hypoperfusion was revealed by SPECT especially in the left cerebral hemisphere (Fig. 1C). Angiography revealed severe stenosis steno-occlusive lesion at the terminal portion of the ICA and horizontal portion of the middle cerebral artery (Fig. 1D-F). Moyamoya vessels were significantly developed bilaterally. He underwent EDAS bilaterally and disease progression has not been observed. However, he needs some supports on his daily life because of mental retardation.

\section{Patient 3}

A 6-year-old boy visited our hospital because of right hemiparesis. He had been followed by pediatrician for left arm hypertrophy and mental retardation. Thus, his distinctive face indicated Proteus syndrome (Fig. 2A). CT demonstrated right thalamic hemorrhage with left frontal and right parietal infarction (Fig. 2B). The lesions 
confirmed with MRI and left frontal lesion was revealed as fresh lesion (Fig. 2C, D). Cerebral perfusion was impaired bilaterally (Fig. 2E). Angiography showed steno-occlusive lesion at the terminal portion of the bilateral ICA (Fig. 2F, G). Moyamoya vessels were massively developed especially in the right side. Posterior cerebral arteries were also involved (Fig. 2H). Collateral flow from external carotid artery (ECA) was slightly seen (Fig. 2I, J). STA-MCA anastomosis combination with EMS was performed bilaterally. Postoperative course was uneventful. However, he need some supports on his daily life because of mental retardation.

\section{Patient 4}

A-5-year-old boy, who has a history of partial removal of left temporal glioma with local irradiation, presented because of incidentally found cerebral infarction. MRI showed right frontal infarction as well as residual tumor in the left temporal lobe (Fig. 3A). SPECT revealed hypoperfusion in the right frontal and left parietal lobe (Fig. 3B). Steno-occlusive lesion was found at the terminal portion of the bilateral ICA and moyamoya vessels were developed (Fig. 3C-E). Collateral flow from ECA was significantly developed (Fig. 3F, G). Reoperation was performed for the recurrence of the tumor. He was followed clinically and radiologically. Additional ischemic lesion has not been demonstrated. However, his daily life was affected by mental retardation. 


\section{Discussion}

\section{Patient Characteristics}

According to the diagnostic criteria of the RCMJ, the etiology of moyamoya disease is unknown and the condition associated with atherosclerosis, autoimmune disease, meningitis and so on, are distinguished from definite moyamoya disease $[4,5]$. Since the terminology for this condition has not been defined, several words have been used to mean this condition as quasi-moyamoya disease, akin-moyamoya disease, moyamoya syndrome, moyamoya phenomenon, moyamoya-like vasculopathy and so on $[1,3,6,7]$. We employed the term "quasi-moyamoya disease" throughout this report because it was given by Dr. Suzuki, who reported "moyamoya disease" at first [5]. This incidence of quasi-moyamoya disease $(7 / 69=10.8 \%)$ was consistent with previous Japanese reports, which demonstrated approximately $10 \%$ of moyamoya disease [8]. The ratio of quasi-moyamoya disease is higher in western country [7]. Among seven patients of moyamoya syndrome, five were pediatric patients. Table 1 provides a summary of the clinical data obtained from the five quasi-moyamoya disease patients. The mean age was 6.4-year-old. It is well known that patients with moyamoya disease are predominantly female and we have demonstrated that the moyamoya syndrome affected patients are predominantly male. In terms of patient ages, it has been reported that there are two peaks, namely child type and adult type $[9,10]$. In this series, child types are dominant because of congenital disorder or pediatric-onset brain tumor. 


\section{Symptoms and Sings}

The clinical manifestation of quasi-moyamoya disease are complicated since the aspect of cerebrovascular disorder and that of associated disorder are merged. Interestingly, mental retardation was seen in the most congenital disorder. Regarding with cerebrovascular disorder, four patients suffered ischemia and one patient had both hemorrhage and ischemia. As shown in Fig. 2B, the hemorrhage was seen in the right thalamus, which was unusual as moyamoya disease.

\section{Radiological findings}

We identified a wide variety of angiographical lesions in our series. All patients had arterial steno-occlusion with formation of moyamoya vessels bilaterally. Moyamoya disease associated with Down syndrome or neurofibromatosis type 1 occasionally involves only unilateral side [1, 11]. Moyamoya vessels were well developed in congenital disorders and were not significant in irradiated brain tumor patients. Collateral flow form ECA was significantly dominant in irradiated brain tumor patients. [12]. Posterior circulation was involved in three patients. Taken together, angiographical finding of quasi-moyamoya disease is widely varied.

\section{Treatment and prognosis}

In terms of treatment of quasi-moyamoya disease, vascular recanalization was performed for the patient with symptom or cerebral hypoperfusion revealed by SPECT. Two patients underwent STA-MCA direct bypass procedure. Indirect vascular 
reconstruction was performed to two patients. Postoperative course was uneventful. Thus, vascular reconstruction was effective to prevent disease progression and cerebral hypoperfusion was improved postoperatively. Moyamoya disease, particularly in children, often exhibit progression during follow-up. Among our quasi-moyamoya disease patients, angiographical progression was seen in two irradiated brain tumor patients. In these two patients, vascular reconstruction was not employed for them, since collateral flow from ECA had been developed. Finally, activity of daily living of the patients was influenced with symptoms such as mental retardation due to associated disorder. And one patient died of brain tumor recurrence.

\section{Genetic analysis}

The pathologic and epidemiological facts suggest that genetic factors play a more important role in the pathogenesis of moyamoya disease than do acquired factors [13]. The familial occurrence was approximately $10 \%$ of moyamoya disease patients $[14,15]$. Previous linkage studies have indicated that susceptibility loci for the moyamoya disease are located on chromosome 3p24.2-26, 6q, 8q23 and 17q25 [13, 16-18]. Mineharu et al reported that the mode of inheritance of familial moyamoya disease is autosomal dominant with incomplete penetrance [19]. Several genetic factors in different loci cause the same disease (locus heterogeneity). Thus, the coincidence of unilateral and definite moyamoya disease within a single family indicates that they reflect different phenotypes cause by the same genetic defects [20]. Some of the congenital disorders namely neurofibromatosis type 1 , of which responsible gene is 
located on chromosome 17q11.2 may be related to the same gene with moyamoya disease. Others such as Down syndrome are cased by completely different gene, but manifest similar cerebral vascular disease.

\section{Limitation}

The incidence of moyamoya disease is estimated to be 0.54 per 100.000 populations and that of quasi- moyamoya disease is as low as $10 \%$ of moyamoya disease [21]. Therefore, it is difficult to demonstrate clinical feature of quasi- moyamoya disease. Nation wide study is required to clarify the clinical feature of quasi- moyamoya disease and propose proper treatment. 


\section{Conclusions}

Among five cases of quasi-moyamoya disease, no one exhibited typical clinical course of moyamoya disease. Clinical manifestation and pathophysiology of quasi-moyamoya disease were diverse according to their associated disorders. Vascular reconstruction prevented disease progression and the prognosis of the quasi-moyamoya disease was influenced with associated disorders. 


\section{References}

1) Horn P, Pfister S, Bueltmann E, Vajkoczy P, Schmiedek P. Moyamoya-like vasculopathy (moyamoya syndrome) in children. Childs Nerv Syst. 2004;20:382-391

2) Jea A, Smith ER, Robertson R, Scott RM. Moyamoya syndrome associated with Down syndrome: outcome after surgical revascularization. Pediatrics. 2005;116:e694-701

3) Kestle JR, Hoffman HJ, Mock AR. Moyamoya phenomenon after radiation for optic glioma. J Neurosurg. 1993;79:32-35

4) Fukui M. Guidelines for the diagnosis and treatment of spontaneous occlusion of the circle of Willis ('moyamoya' disease). Research Committee on Spontaneous Occlusion of the Circle of Willis (Moyamoya Disease) of the Ministry of Health and Welfare, Japan. Clin Neurol Neurosurg. 1997;99 Suppl 2:S238-S240

5) Suzuki J. Quasi-Moyamoya diseases. In: Suzuki J, eds. Moyamoya disease. New York: Springer-Verlag; 1986:145-168

6) Hallemeier CL, Rich KM, Grubb RL Jr, Chicoine MR, Moran CJ, Cross DT 3rd, Zipfel GJ, Dacey RG Jr, Derdeyn CP. Clinical features and outcome in North American 
adults with moyamoya phenomenon. Stroke. 2006:37:1490-1496

7) Scott RM, Smith JL, Robertson RL, Madsen JR, Soriano SG, Rockoff MA. Long-term outcome in children with moyamoya syndrome after cranial revascularization by pial synangiosis. J Neurosurg. 2004;100(2 Suppl Pediatrics):142-149

8) Komiyama M, Nakajima H, Nishikawa M, Yasui T, Kitano S, Sakamoto H, Fu Y. High incidence of persistent primitive arteries in moyamoya and quasi-moyamoya diseases. Neurol Med Chir (Tokyo). 1999;39:416-420

9) Fukui M. Current state of study on moyamoya disease in Japan. Surg Neurol. $1997 ; 47: 138-143$

10) Wakai K, Tamakoshi A, Ikezaki K, Fukui M, Kawamura T, Aoki R, Kojima M, Lin Y, Ohno Y. Epidemiological features of moyamoya disease in Japan: findings from a nationwide survey. Clin Neurol Neurosurg. 1997;99 Suppl 2:S1-S5

11) Rosser TL, Vezina G, Packer RJ. Cerebrovascular abnormalities in a population of children with neurofibromatosis type 1. Neurology. 2005;64:553-555

12) Ishikawa $T$, Houkin $K$, Yoshimoto $T$, Abe $H$. Vasoreconstructive surgery for 
radiation-induced vasculopathy in childhood. Surg Neurol 1997;48:620-626

13) Yamauchi T, Tada M, Houkin K, Tanaka T, Nakamura Y, Kuroda S, Abe H, Inoue T, Ikezaki K, Matsushima T, Fukui M. Linkage of familial moyamoya disease (spontaneous occlusion of the circle of Willis) to chromosome 17q25. Stroke. 2000;31:930-935

14) Fukui M, Kono S, Sueishi K, Ikezaki K. Moyamoya disease. Neuropathology. 2000;20 Suppl:S61-S4

15) Nanba R, Kuroda S, Tada M, Ishikawa T, Houkin K, Iwasaki Y. Clinical features of familial moyamoya disease. Childs Nerv Syst. 2006;22:258-262

16) Ikeda H, Sasaki T, Yoshimoto $T$, Fukui M, Arinami T. Mapping of a familial moyamoya disease gene to chromosome 3p24.2-p26. Am J Hum Genet. 1999;64:533-537

17) Inoue TK, Ikezaki K, Sasazuki T, Matsushima T, Fukui M. Linkage analysis of moyamoya disease on chromosome 6. J Child Neurol. 2000;15:179-182

18) Sakurai K, Horiuchi Y, Ikeda H, Ikezaki K, Yoshimoto T, Fukui M, Arinami T. A novel susceptibility locus for moyamoya disease on chromosome 8q23. J Hum Genet. 
2004;49:278-281

19) Mineharu Y, Takenaka K, Yamakawa H, Inoue K, Ikeda H, Kikuta KI, Takagi Y, Nozaki K, Hashimoto N, Koizumi A. Inheritance pattern of familial moyamoya disease: autosomal dominant mode and genomic imprinting. J Neurol Neurosurg Psychiatry. 2006;77:1025-1029

20) Kusaka N, Tamiya T, Adachi Y, Katayama S, Namba S, Tokunaga K, Sugiu K, Date I, Ohmoto T. Adult unilateral moyamoya disease with familial occurrence in two definite cases: a case report and review of the literature. Neurosurg Rev. 2006;29:82-87

21) Kuriyama S, Kusaka Y, Fujimura M, Wakai K, Tamakoshi A, Hashimoto S, Tsuji I, Inaba Y, Yoshimoto T. Prevalence and clinicoepidemiological features of moyamoya disease in Japan: findings from a nationwide epidemiological survey. Stroke. 2008;39:42-7. 


\section{Figure Legends}

\section{Fig. 1 Patient 1}

A 6-year-old boy with Saethre-Chotzen syndrome.

Three-dimensional skull bone CT showed craniosynostosis (A). MRI (T2 weighted image) showed multiple lacuna infarctions (B). Cerebral hypoperfusion was revealed by single photon emission tomography (SPECT) especially in the left frontal lobe (C). Angiography reveal steno-occlusive lesion at the terminal portion of the bilateral internal carotid artery (ICA; D, E). Moyamoya vessels were also developed bilaterally. Posterior circulation was not involved (F).

\section{Fig. 2 Patient 3}

A 6-year-old boy with Proteus syndrome.

Distinctive face with left arm hypertrophy were seen (A). CT demonstrated right thalamic hemorrhage with left frontal and right parietal infarction (B), and lesions were confirmed by MRI (inversion recovery image; C). MRI (diffusion-weighted image) showed left frontal lesion as fresh lesion (D). Cerebral perfusion was impaired bilaterally (E). Angiography showed steno-occlusive lesion at the terminal portion of the bilateral ICA (F, G). Moyamoya vessels were developed especially in the right side. Pial anastomosis from the left posterior cerebral artery was developed (H). Collateral flow from external carotid artery was slightly seen (I, J). 


\section{Fig. 3 Patient 4}

A-5-year-old boy with left temporal glioma and cranial irradiation.

MRI (inversion recovery image) showed residual tumor in the left temporal lobe as well as right frontal infarction (A). SPECT revealed hypoperfusion in the right frontal and left parietal lobe (B). Steno-occlusive lesion was found at the terminal portion of the bilateral ICA and moyamoya vessels were developed (C, D). Pial anastomosis from the right posterior cerebral artery was developed (E). Collateral flow from ECA was significantly developed (F, G). 
Fig. 1
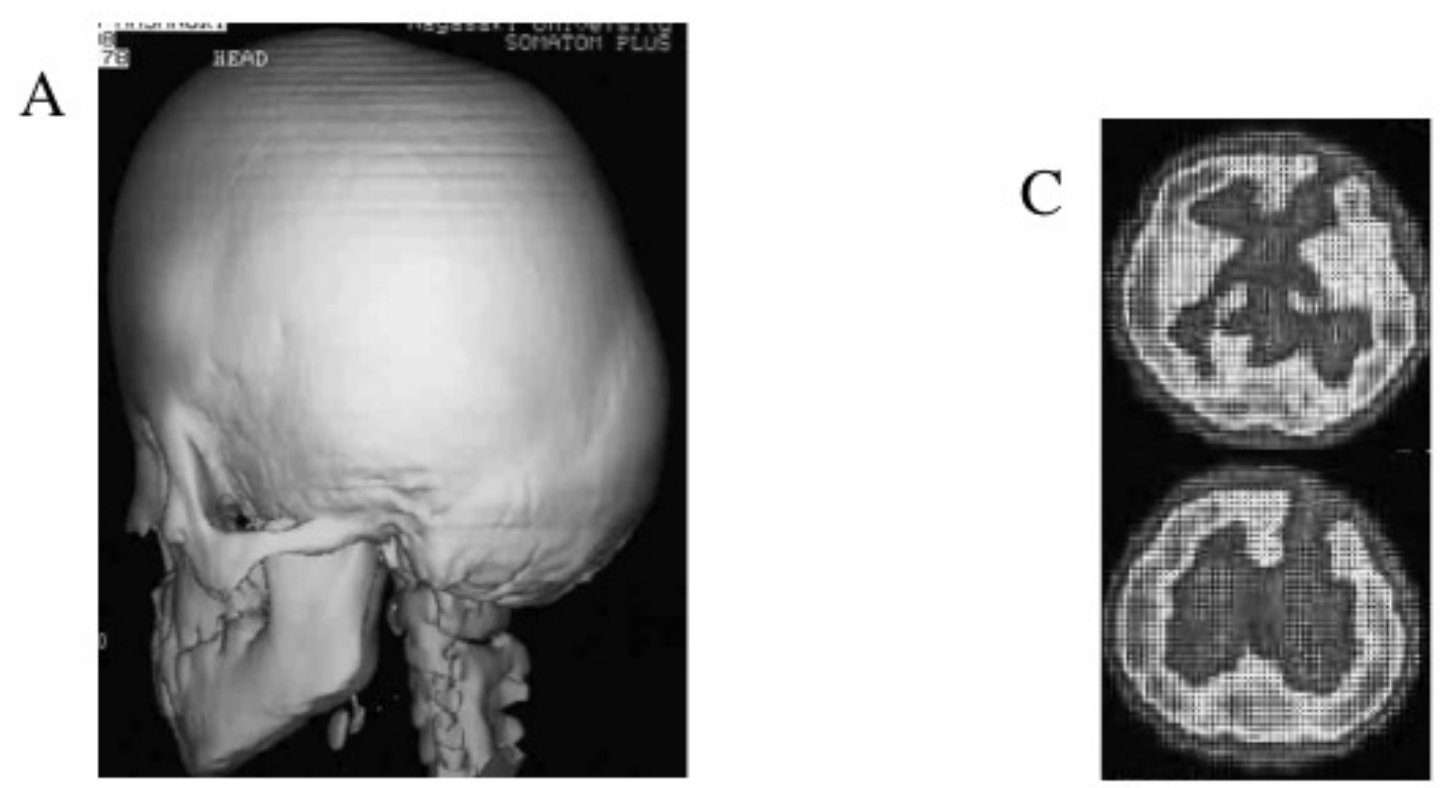

B

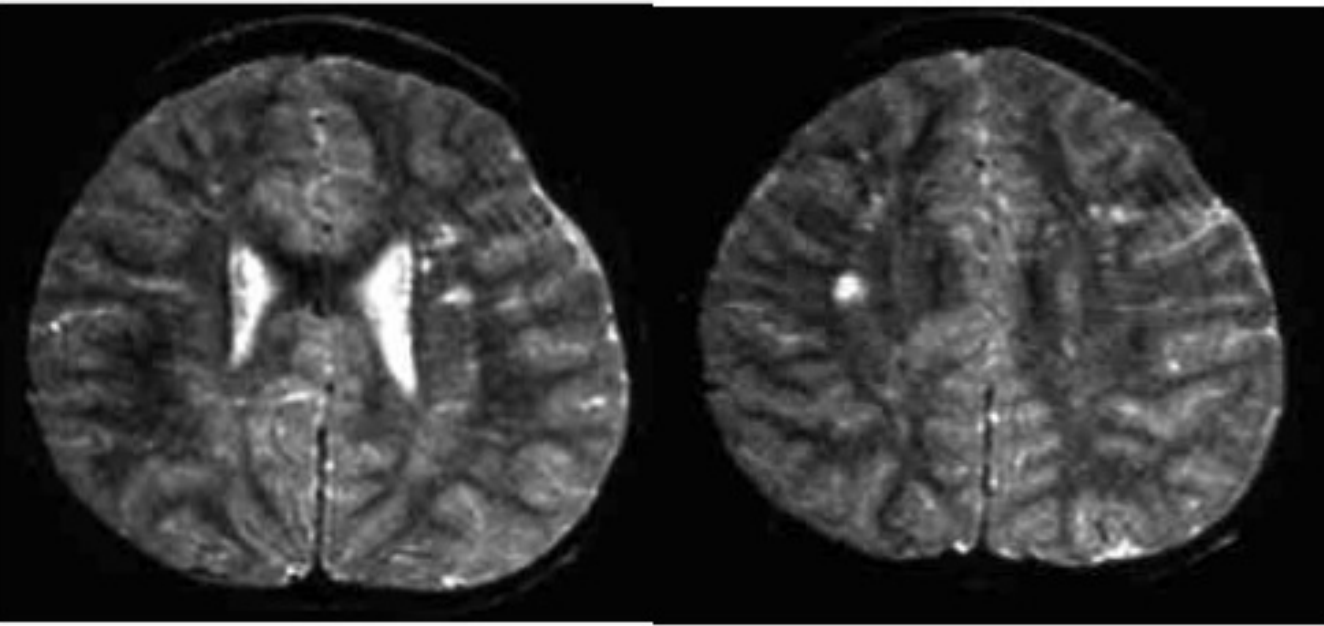


Fig. 1
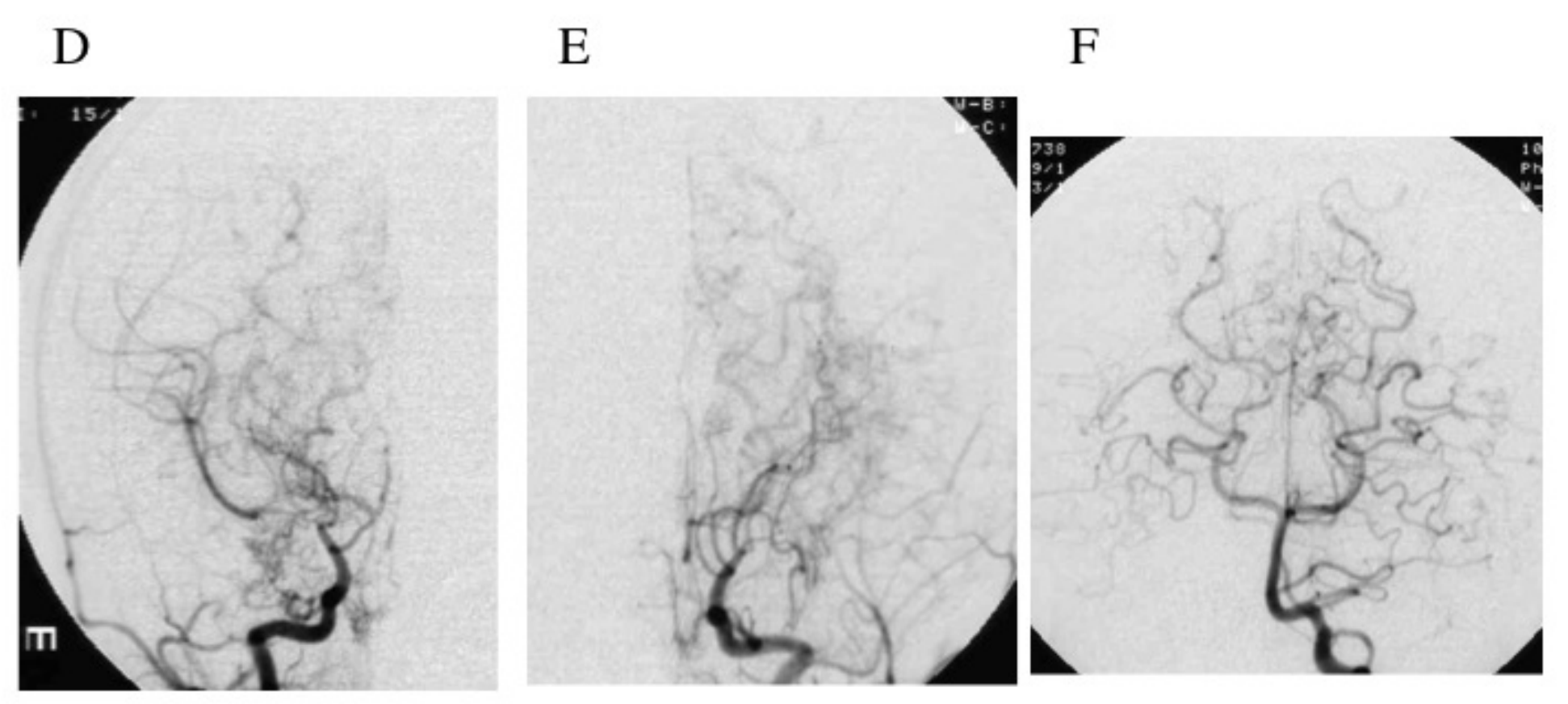
Fig. 2 A

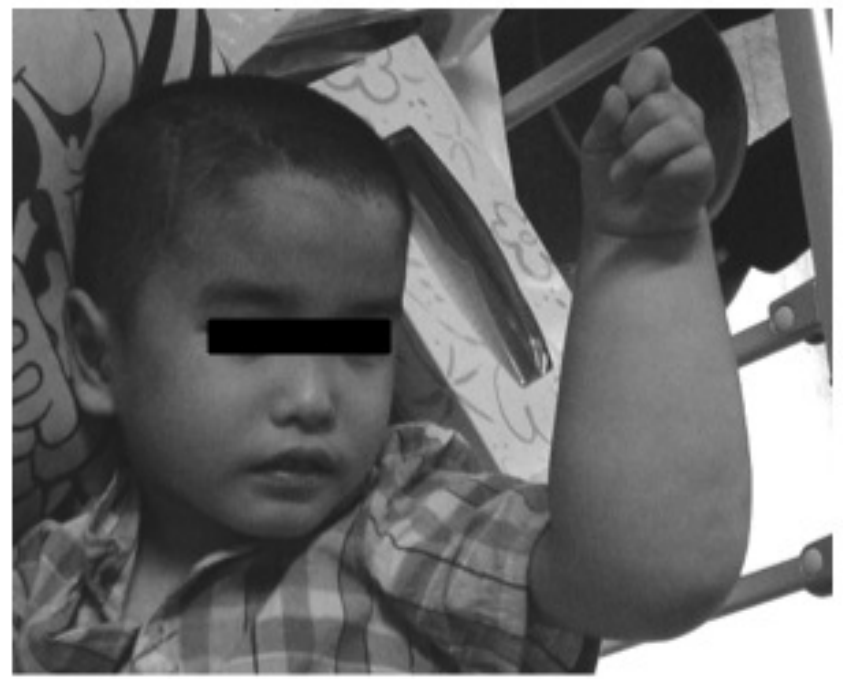

B

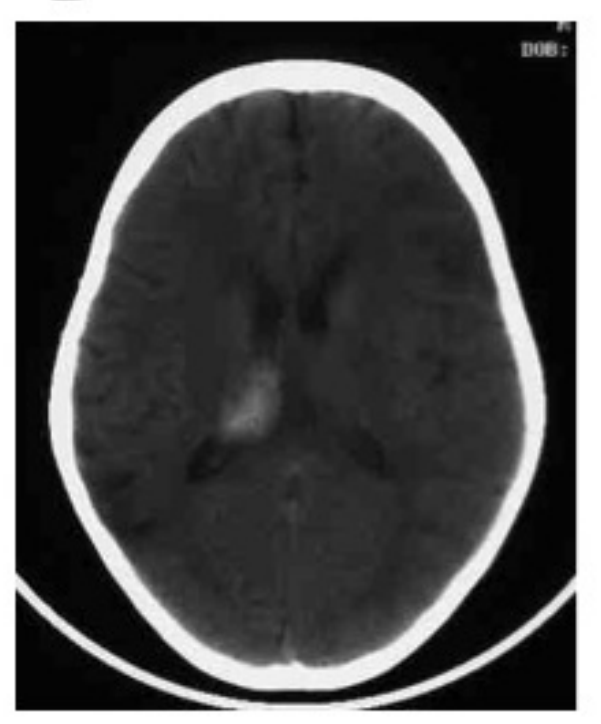

C

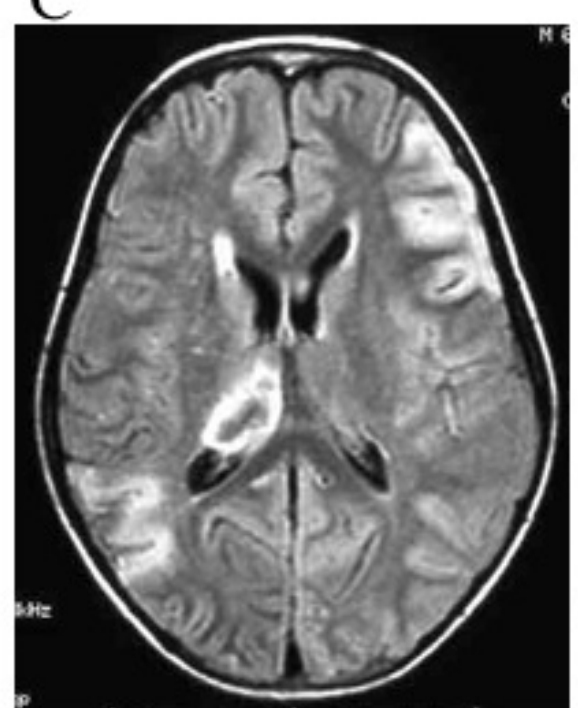

D

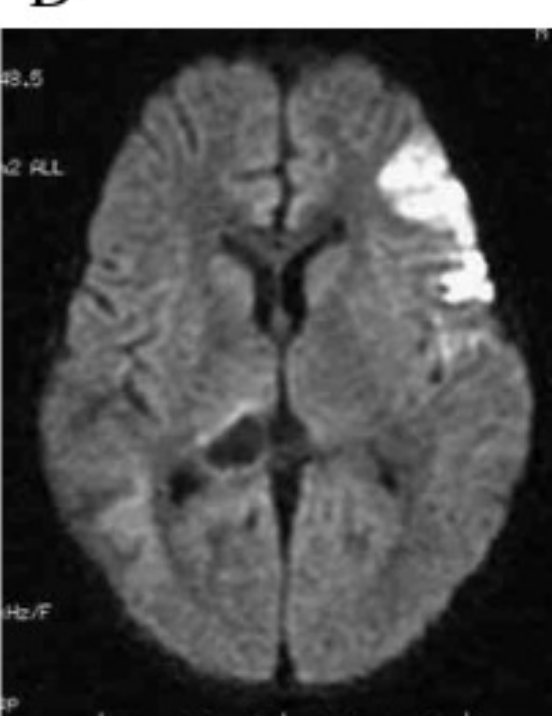

E

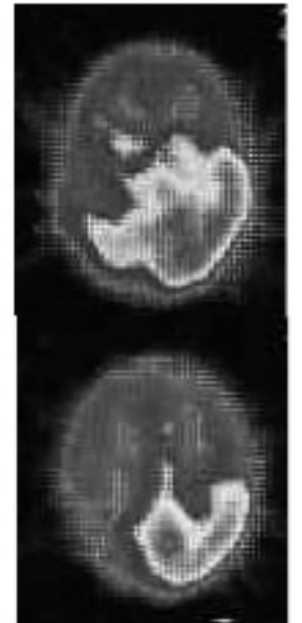




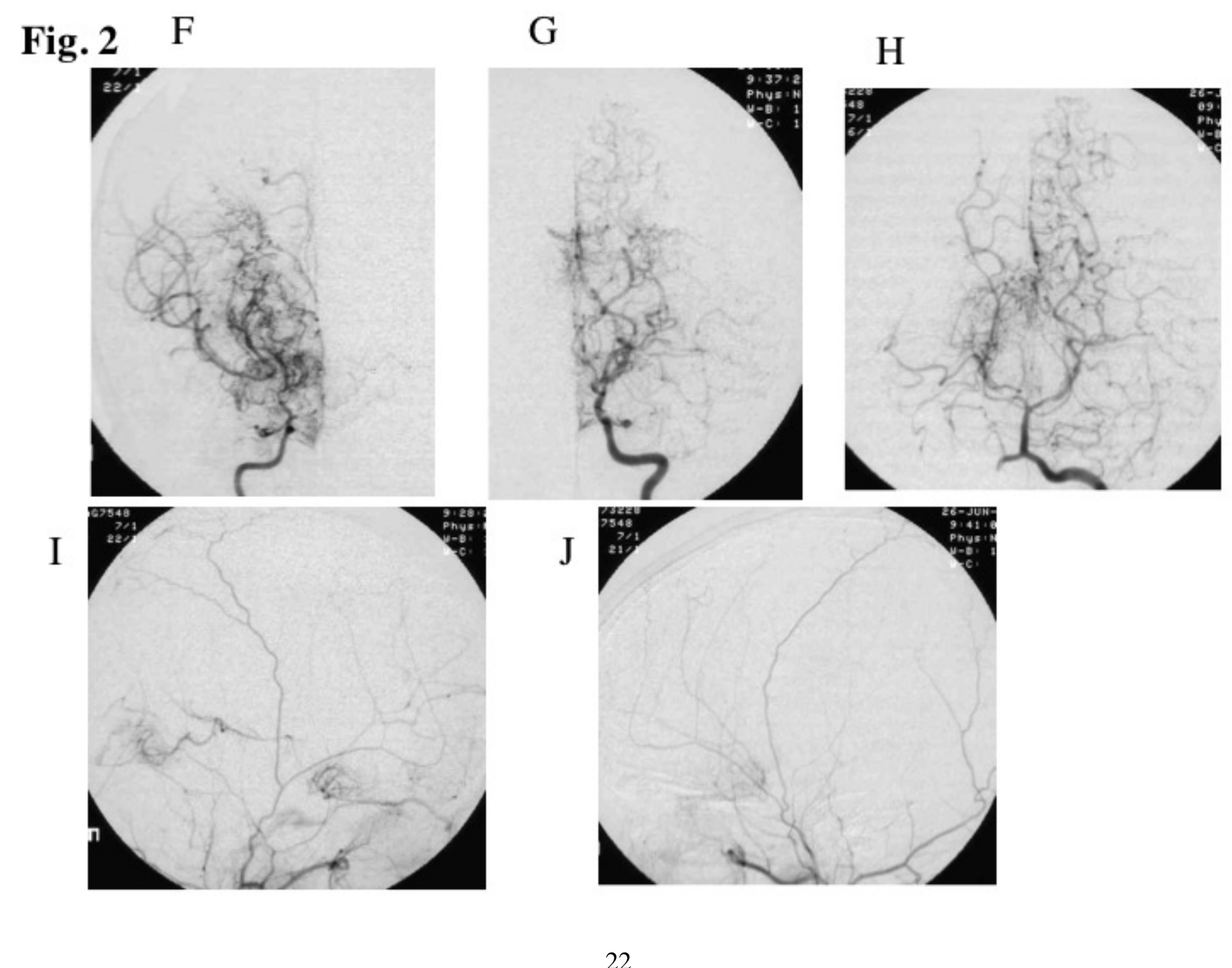


Fig. 3

A

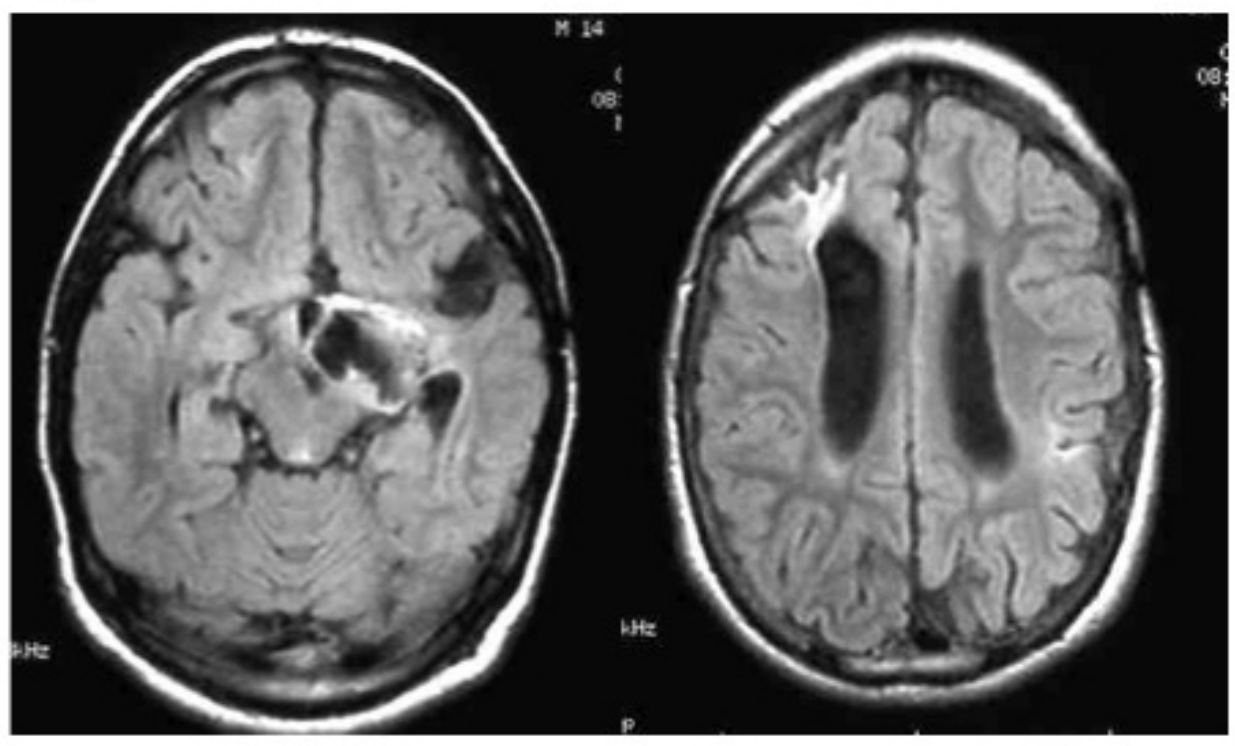

B

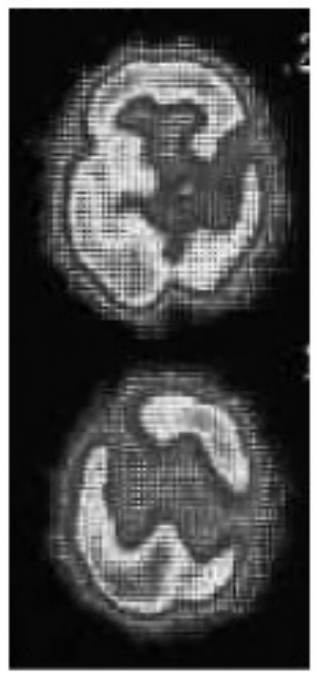



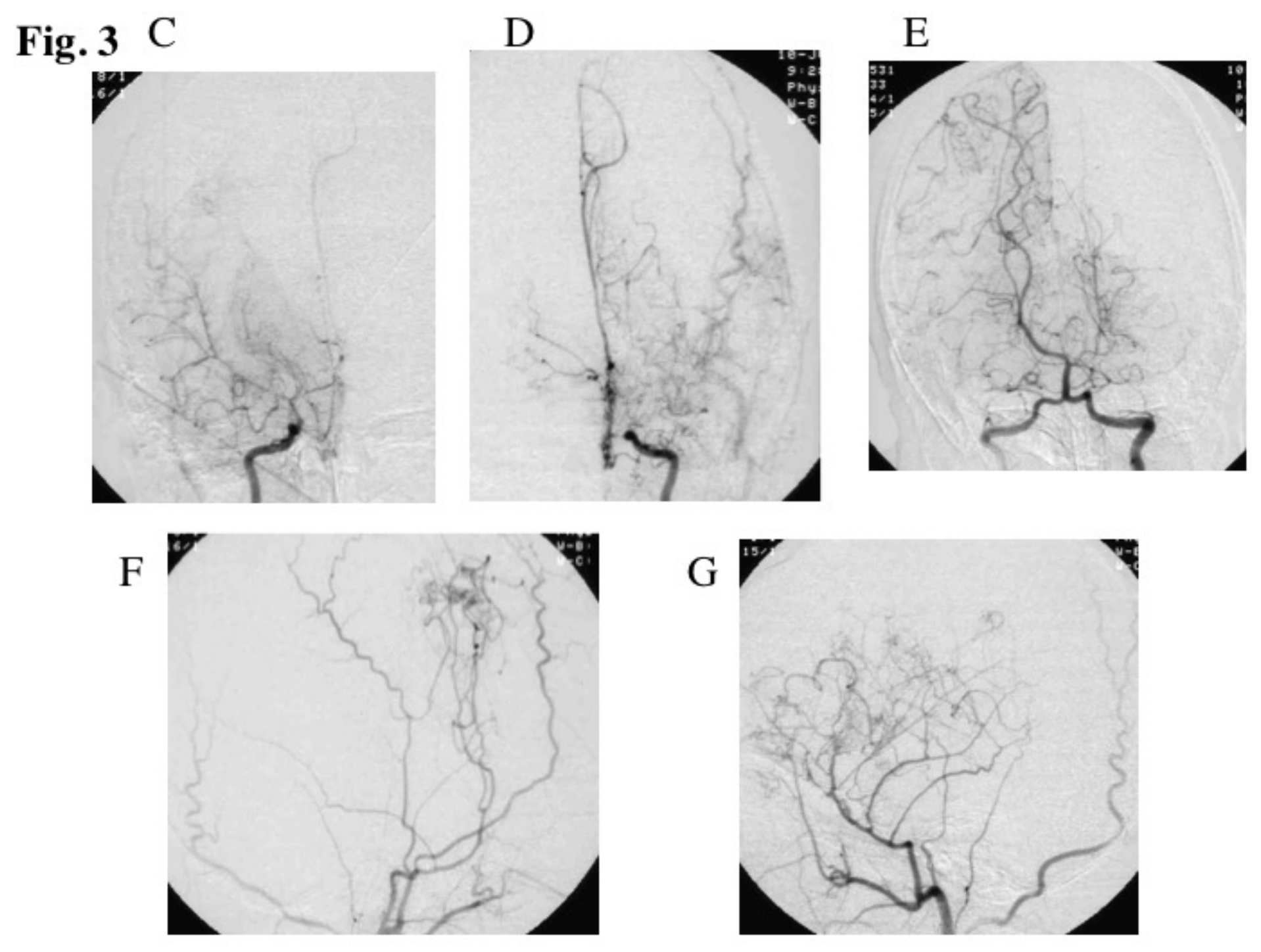
Table 1 patient characteristics of quasi-moyamoya disease

Age, sex disease symptom

imaging study

treatment

follow (years)

1) $6 \mathrm{M}$ craniosynostois

gate disturbance

cerebral infarction

Bil. EDAS

14

(mental returdation)

2) $9 \mathrm{M}$ dwarfism

epilepsy

no abnormality

Bil. EDAS

coarctation of aorta

3) $6 \mathrm{M}$ Proteus syndrome TIA

(mental returdation)

cerebral infarction

Bil. STA-MCA

intracerebral hemorrhge

with EMS

cerebral infarction

(removal)

(brin tumor)

5) $6 \mathrm{~F} \quad \mathrm{BT} /$ irradiation (visual disturbance)

(mental returdation)

cerebral infarction

(removal)

5 (dead)

(brain tumor)

M: male, F: female, NF: neurofibromatosis type 1, BT: brain tumor, TIA: transient ischemic attack, Bil: bilateral EDAS: encephalo-duro-arterio-synostosis, STA-MCA: superficial temporal artery-middle cerebral artery anastomosis ( ) indicates findings due to associated disorder or treatment for the disorder. 